\title{
Prótese parcial removível temporária em Odontopediatria: relato de caso
}

\author{
Temporary removable partial denture in Pediatric Dentistry : case report \\ Prótesis parcial removible temporal en Odontología Pediátrica: reporte de caso \\ Keicy Louene de Almeida Teixeira BRELAZ ${ }^{\mathbf{1}}$ \\ Gisely Naura VENÂNCIO ${ }^{2}$ \\ Mailza Costa de ALMEIDA ${ }^{3}$ \\ Carolina Rocha AUGUSTO ${ }^{4}$
}

${ }^{l}$ Departamento de Dentística e Prótese, Faculdade de Odontologia, UniNilton Lins, Universidade Nilton Lins.

Av. Professor Nilton Lins, 3259. Parque das Laranjeiras, 69058-030, Manaus-AM, Brasil. keicylouene@yahoo.com.br

${ }^{2}$ Departamento de Dentística e Prótese, Faculdade de Odontologia, UniNilton Lins, Universidade Nilton Lins.

Av. Professor Nilton Lins, 3259. Parque das Laranjeiras, 69058-030, Manaus - AM, Brasil. (92) 991764966. ginaura@gmail.com

${ }^{3}$ Faculdade de Odontologia, Universidade Federal do Amazonas,

Rua Ministro Waldemar Pedrosa, 1539, Praça 14 de Janeiro, 69025-050, Manaus - AM, Brasil. mailzalmeida@gmail.com

${ }^{4}$ Departamento de Materiais Dentários e Prótese, Faculdade de Odontologia, Universidade do Estado do Amazonas.

Av. Carvalho Leal, 1777, Cachoeirinha,69065-001, Manaus-AM, Brasil. carolinarochaaugusto@ gmail.com

\section{Resumo}

A perda precoce dos elementos dentais anteriores em crianças influencia o comportamento psicossocial infantil. As próteses temporárias devolvem a aparência estética até que uma prótese definitiva possa ser confeccionada, além de ser importante para a manutenção do espaço. Este trabalho tem como objetivo apresentar um caso de reabilitação bucal com a confecção de prótese parcial removível temporária na arcada superior de um paciente de 12 anos de idade, gênero masculino, atendido na Clínica de Graduação de Odontopediatria de instituição privada, insatisfeito com seu sorriso devido à fratura coronária dos incisivos centrais superiores e posterior perda precoce de dentes permanentes. Os resultados demonstraram que o tratamento reabilitador foi resolutivo para repor os elementos perdidos, atuando na preservação do espaço, sem interferir no crescimento ósseo, sendo necessário até a estabilização da dimensão vertical de oclusão, impedindo o fechamento das regiões edêntulas, restabelecendo as principais funções orais e devolvendo a estética e a autoestima.

Descritores: Prótese Dentária; Prótese Parcial Temporária; Mantenedor de Espaço; Estética.

\begin{abstract}
The early loss of previous dental elements in children influences the children's psychosocial behavior. The temporary prostheses return the aesthetic appearance until a permanent prosthesis can be made in addition to being important for the maintenance space. This work aims to present a case of oral rehabilitation after early loss of permanent teeth with temporary removable partial denture in the upper arch of a 12-year-old patient, male gender, attended at the Pediatric Dentistry Graduate Clinic of a private institution, dissatisfied with his smile due to coronary fracture of maxillary central incisors and subsequent early loss of permanent teeth. The results demonstrated that the rehabilitation treatment was resolute to replace the missing elements, acting on the preservation of space, without interfering with bone growth, being necessary until stabilization of the occlusal vertical dimension, preventing the closing of the edentulous regions, restoring the oral main functions and restoring aesthetics and self-esteem.
\end{abstract}

Descriptors: Dental Prosthesis; Denture, Partial, Temporary; Space Maintenance; Esthetics.

\section{Resumen}

La pérdida temprana de elementos dentales anteriores en los niños influye en el comportamiento psicosocial de los niños. Las prótesis temporales vuelven apariencia estética hasta una prótesis permanente puede hacerse además de ser importante para el espacio de mantenimiento. Este estudio tiene como objetivo presentar un caso de la rehabilitación oral con la confección de prótesis parcial removible temporal en la mandíbula superior de un paciente de 12 años de edad, sexo masculino, asistió a la institución privada de Odontología Pediátrica Clínica de Posgrado, insatisfechos con su sonrisa debido a la fractura coronaria de los incisivos centrales superiores y pérdida temprana posterior de los dientes permanentes. Estos resultados demuestran que el tratamiento de rehabilitación estaba resolviendo para restaurar los elementos perdidos, que actúa sobre el espacio de conservación, sin interferir con el crecimiento del hueso que se necesita para estabilizar la dimensión vertical de la oclusión, lo que impide el cierre de las regiones desdentados, restaurando el principal funciones orales y la estética y la restauración de la autoestima.

Descriptores: Prótesis Dental; Dentadura Parcial Provisória; Mantenimiento del Espacio; Estética. 


\section{INTRODUÇÃO}

A perda precoce dos elementos dentais anteriores frequentemente influencia o comportamento social de crianças e adolescentes, além de trazer problemas funcionais e estéticos, podendo comprometer o bem-estar psíquico-social do indivíduo ${ }^{1}$.

A reposição protética dos elementos dentários por meio de mantenedores de espaço visa devolver a integridade das arcadas, restabelecendo as funções normais como mastigação, deglutição e fonação, evitando a instalação de hábitos nocivos e maloclusões ${ }^{2,3}$.

Uma prótese parcial removível pode apresentar desafios com relação à correspondência de cor, tamanho e forma, e que muitas vezes requer a modificação substancial para alcançar uma aparência aceitável ${ }^{4}$.

A reabilitação estética e funcional de pacientes com dentes muito desgastados e com edentulismo parcial é um desafio que exige habilidade e conhecimento técnico pelo dentista, havendo tais problemas múltiplas implicações para o paciente em relação à perda de guia anterior, redução da dimensão vertical de oclusão (DVO) e desfiguração oclusal ${ }^{5}$.

Shibayama et al. ${ }^{6}$ (2006) descrevem que muitos pacientes não suportam a situação de permanecerem desdentados após as extrações dos dentes remanescentes, em virtude do tempo necessário para a completa cicatrização e reparação óssea. No entanto, além do fator psicológico, outros fatores como estética, fonética, mastigação e convívio social estão envolvidos em tal situação.

Segundo Dantas ${ }^{7}$ (2012), o restabelecimento da DVO é de extrema importância para o sucesso das reabilitações bucais, pois se não for restabelecida corretamente, poderá causar danos nos dentes, músculos, articulação têmporo-mandibular (ATM) e sistema auditivo, na deglutição e fonação, e até mesmo na postura do paciente, podendo afetar seu equilíbrio.

Os mantenedores podem ser fixos ou removíveis; os primeiros são confeccionados em aço inoxidável e fixados aos dentes e os removíveis são confeccionados em resina acrílica, podendo ter um parafuso expansor, que pode ser periodicamente ajustado. A indicação depende de certos requisitos como quantidade de elementos dentários perdidos, tipo de oclusão dentária, faixa etária do paciente e seu grau de colaboração. Essas próteses também devem ser desgastadas e reembasadas nas consultas periódicas a fim de se evitar o impedimento do crescimento ósseo maxilar nos sentidos latero-lateral e anteroposterior ${ }^{8}$.

Após a perda traumática de um dente anterior, é importante que uma substituição imediata seja realizada, a fim de se evitar dificuldades estéticas, mastigatória e fonética e para manter o espaço desdentado ${ }^{9}$.

Os principais achados clínicos com perda dentária sem reposição são: sobre erupção dos dentes remanescentes, desalinhamento dentário, perda de DVO e desfiguração significativa do plano oclusal ${ }^{10}$.

Em pacientes com baixo poder aquisitivo, pouca exigência estética ou mesmo limitações médicas, a PPRT pode ser uma opção viável ou muitas vezes a única opção de tratamento. Independentemente dos fatores acima mencionados, a todos os pacientes com dentição severamente desgastada, com diminuição da DVO e ausência dentária, deve ser apresentada esta opção de tratamento 5 .

Nessa perspectiva, o objetivo deste estudo é reportar um caso clínico de reabilitação bucal após perda precoce de dentes permanentes anteriores com a confecção de prótese parcial removível temporária (PPRT) na arcada superior de um paciente de 12 anos de idade.

\section{CASO CLÍNICO}

Paciente do gênero masculino, 12 anos, compareceu à Clínica Odontológica da Universidade Nilton Lins-AM, relatando insatisfação com seu sorriso, pois seus dentes estavam fraturados (Figuras 1 e 2), devido a trauma durante jogo de futebol ocorrido há 8 meses.

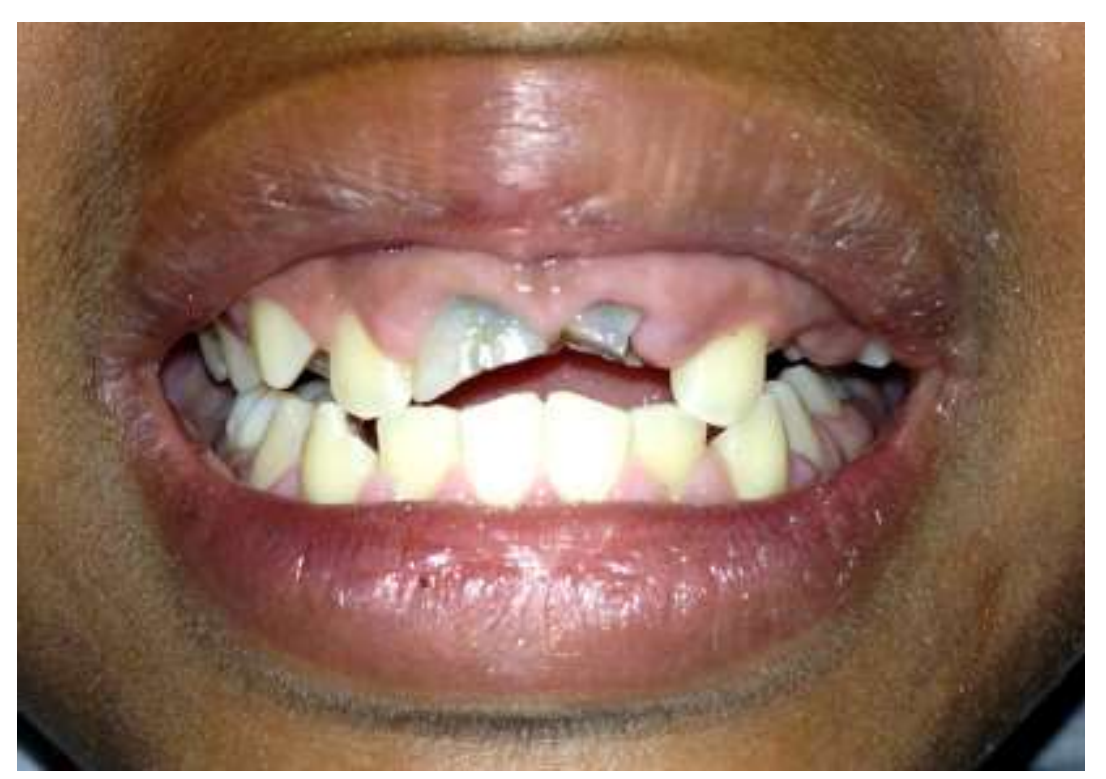

Figura 1. Aspecto intraoral inicial, mostrando os elementos 11 e 21 fraturados

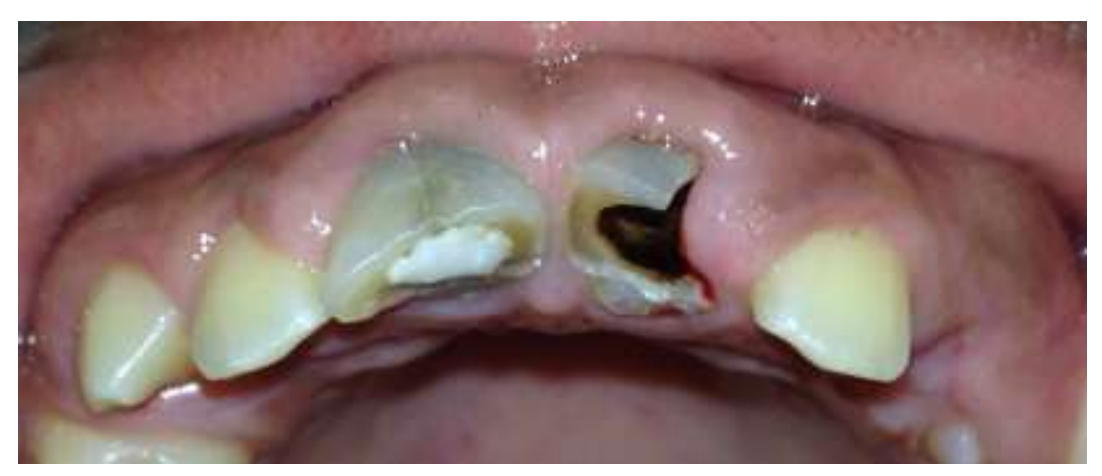

Figura 2. Visão aproximada e por oclusal dos elementos 11 e 21 fraturados 
O responsável pelo paciente relatou que houve uma tentativa de restaurar os elementos dentários realizando tratamento endodôntico para posterior instalação de pinos intrarradiculares e coroas protéticas.

Após anamnese verificou-se que o paciente possuía boa saúde geral. Ao exame físico e radiográfico observaram-se fraturas transversais no terço cervical e médio do elemento 11 e longitudinal do elemento 21 a nível radicular, constatando-se assim a impossibilidade de manutenção dos elementos dentários (Figura 3).

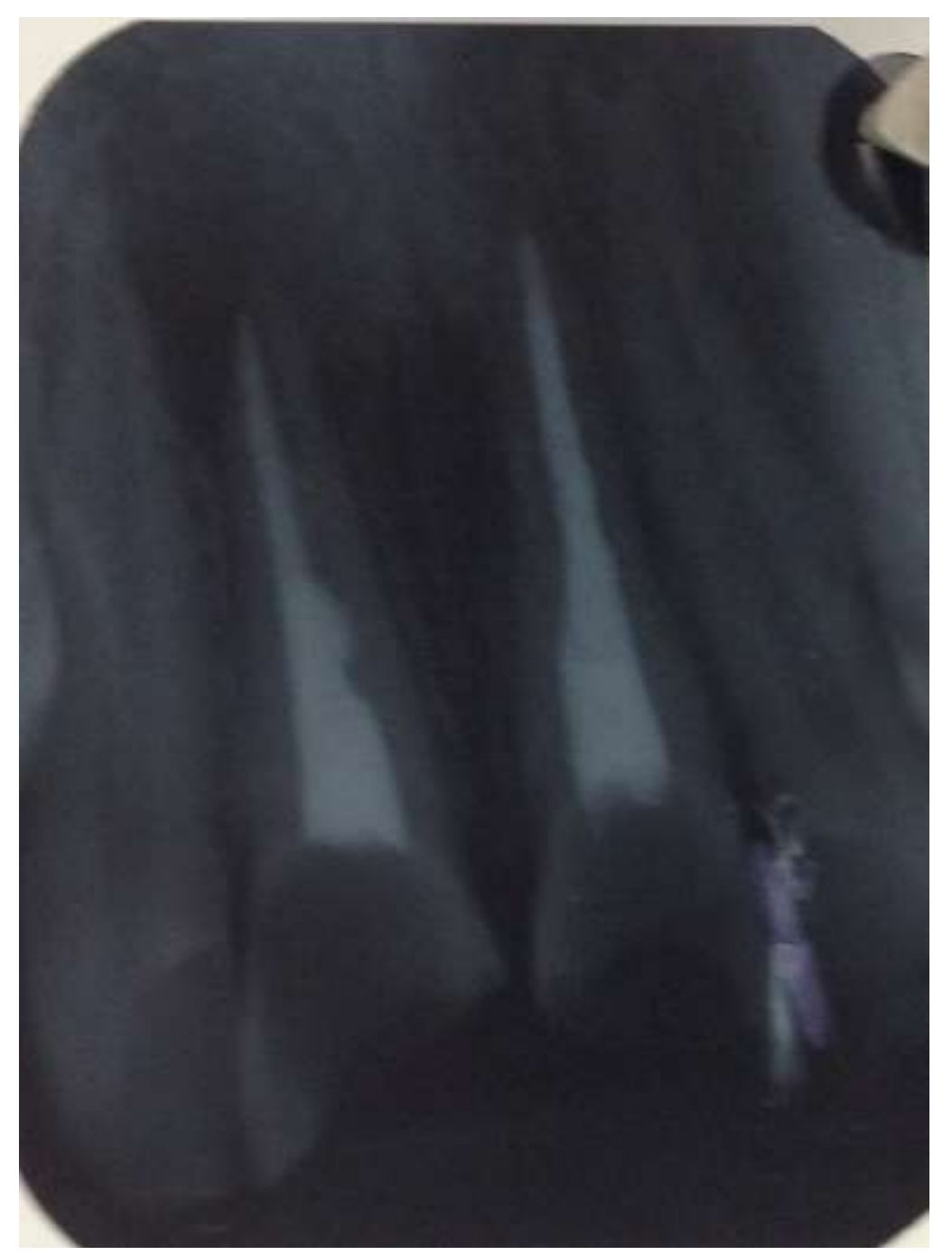

Figura 3. Exame radiográfico mostrando as fraturas transversais no terço cervical e médio do elemento 11 e longitudinal do elemento 21

Após assinatura do termo de consentimento livre e esclarecido (TCLE) pelo responsável do paciente, o tratamento prosposto constituiu em: exodontia dos elementos 11 e 21 com posterior instalação de prótese parcial removível temporária (PPRT), para proporcionar melhor conforto ao paciente.

$\mathrm{Na} 1^{\mathrm{a}}$ sessão, realizou-se a tomada de fotografias intra e extra-orais, seleção e registro de cor, impressão das arcadas superior e inferior, registro de mordida e registro do arco facial. Os modelos obtidos foram montados em articulador semiajustável (ASA), em máxima intercuspidação habitual (MIH), e encaminhados ao laboratório para confecção de enceramento diagnóstico (Figura 4).

$\mathrm{Na} 2^{\mathrm{a}}$ sessão foram realizadas as exodontias e suturas dos elementos 11 e $21 . \mathrm{Na} 3^{\mathrm{a}}$ sessão as sutura foram removidas e, após 30 dias das exodontias (Figura 5), a PPRT foi confeccionada sobre o modelo de trabalho montado em ASA, sendo uma prótese mucosossuportada com reposição dos elementos perdidos em resina acrílica na cor 69 e retenção obtida com grampo de Adams nos elementos 16 e 26 e grampo circunferencial no elemento 14 (Figura 6).

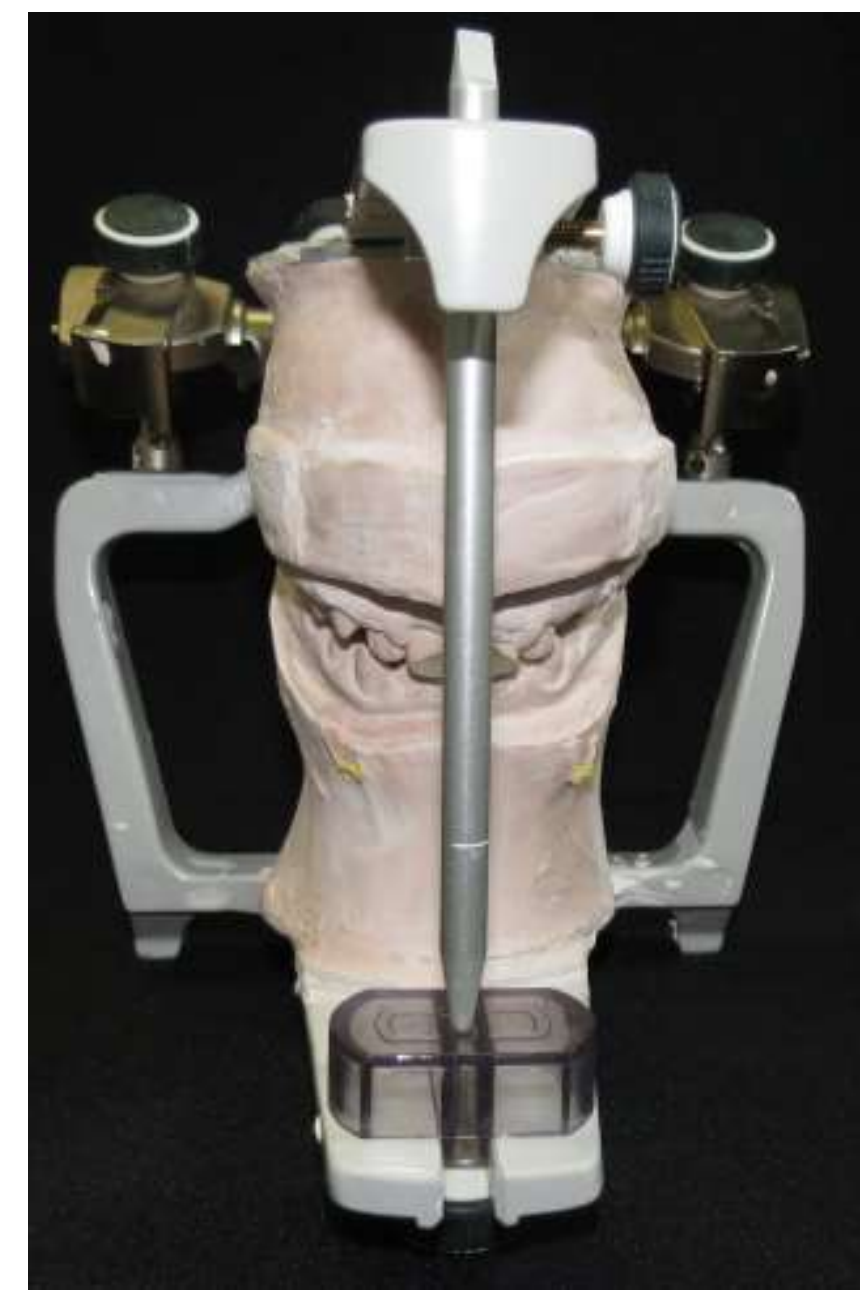

Figura 4. Modelos montados em ASA

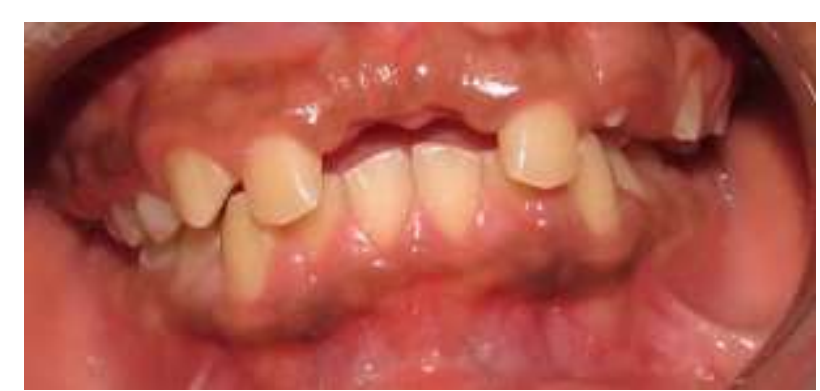

Figura 5. Aspecto intraoral após exodontias dos elementos 11 e 21

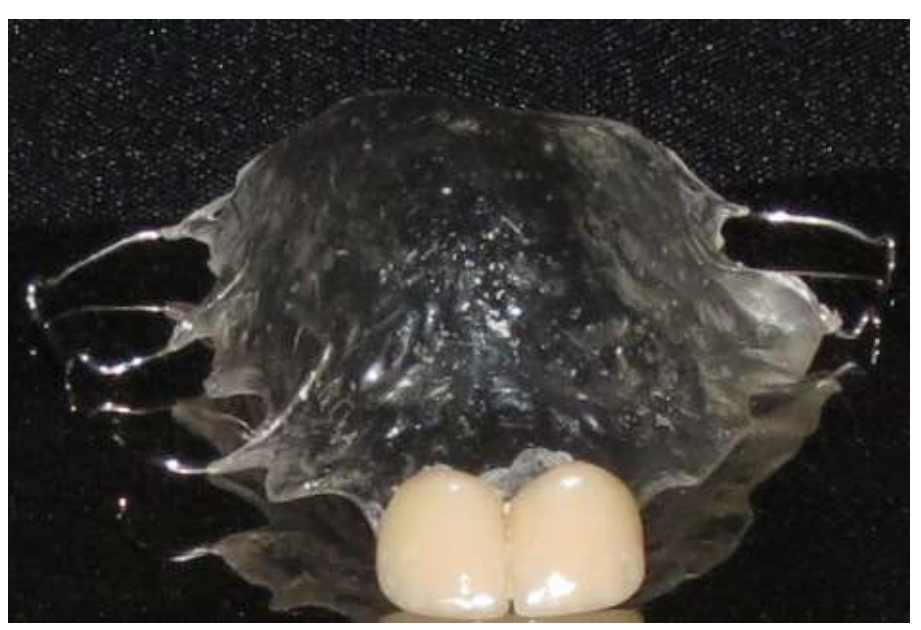

Figura 6. Prótese Parcial Removível Temporária

Durante a instalação da prótese em boca, foi realizado reembasamento com resina acrílica autopolimerizável sob os elementos 11 e 21, seguidos de ajuste oclusal, acabamento e polimento (Figura 7).

As orientações de proservação foram dadas e o paciente foi encaminhado para tratamento $\mathrm{e}$ acompanhamento com Ortodontista para a instalação de aparelho ortodôntico, conscientizando os responsáveis quanto à importância da manutenção 
periódica $^{6}$ até que a DVO esteja completamente definida, com os dentes permanentes erupcionados, para que possa ser confeccionada a prótese definitiva.

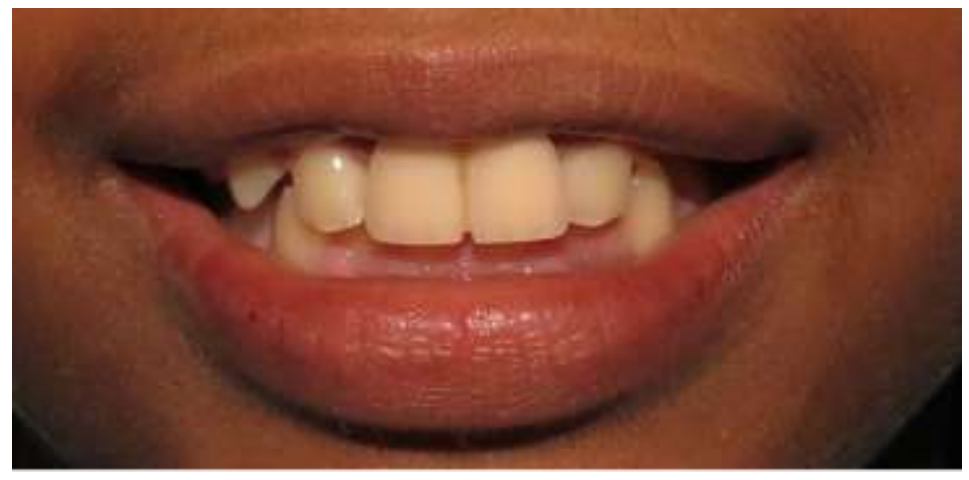

Figura 7. Aspecto intraoral após instalação da prótese

\section{DISCUSSÃO}

A integridade dos dentes é fundamental para a manutenção da oclusão, estética, fonética e bem-estar emocional da criança, adolescente e seus pais, e, nos casos de perda precoce, é considerada como conduta clínica o uso de mantenedores estético-funcionais com reposição dos dentes perdidos precocemente ${ }^{8}$.

Desta forma, a perda de dentes decíduos ou permanentes requer um tratamento combinado abordando tanto estética e função. A metodologia depende da idade do paciente, localização e extensão das lesões traumáticas ${ }^{11}$.

A reabilitação oral Odontopediatria com uma prótese removível é mais complexa e demorada do que em adultos, devendo-se levar em consideração o perfil psicológico e comportamental infantil, a fim de fornecer uma prótese que seja funcional, estética e apropriada para a idade da criança ${ }^{12,13}$.

No caso clínico apresentado, primeiramente foi pensado em um tratamento mais conservador com a manutenção dos elementos 11 e 21, após retratamento endodôntico, instalação de pinos e coroas protéticas, porém ao exame radiográfico verificou-se a fratura a nível radicular em ambos o elementos e, assim, a impossibilidade de manutenção dos mesmos, optandose, portanto, pelas exodontias e confecção de PPRT, devido à idade do paciente.

Prabhakar et al. ${ }^{9}$ (2009) elegem como ideal a substituição imediata dos dentes perdidos para evitar problemas funcionais, de dicção e estéticos, porém, Sander et al. ${ }^{14}$ (2011) e Shibayama et al. ${ }^{6}$ (2006), afirmam que, no caso das próteses removíveis, é necessário um período de tempo após as exodontias, para que haja a completa cicatrização dos tecidos. Portanto, no caso clínico exposto, esperou-se um tempo de 30 dias para a confecção da PPRT, a fim de se evitar o desconforto do paciente.

Pereira e Miasato $^{8}$ (2010) enfatizam que a importância de se restituir elementos dentários através de mantenedores de espaço está fundamentada em estudos que demonstram a alta incidência de fechamento de regiões e extrusões de antagonistas após a perda precoce de dentes decíduos e permanentes.

Segundo Dominguez e Aznar ${ }^{15}$ (2004) e Parisotto et al. ${ }^{16}$ (2009), a reposição dentária dos incisivos centrais superiores é de suma importância para melhorar a fonética, a estética, a deglutição e, principalmente, para evitar a instalação de hábitos como a interposição lingual.

A dentição permanente do paciente era escura, tendo sido escolhida a cor 69 para os dentes da PPRT. Sobre este assunto, Consolaro ${ }^{17}$ (2009) discorre sobre fatores que podem influenciar na cor dos dentes, tais como o meio ambiente do organismo, como produtos derivados da alimentação e medicamentos, as variações de temperatura corporal e os produtos do metabolismo corporal, além dos fatores genéticos.

$\mathrm{Kamble}^{5}$ (2013) descreveu um caso clínico onde foi possível aumentar a DVO e reabilitar estética e funcionalmente o paciente utilizando uma PPRT, para tratar o problema da dentição extremamente desgastada e o edentulismo parcial, adequando corretamente a oclusão do paciente.

Tal sucesso também foi alcançado no caso clínico apresentado, onde a reabilitação bucal com PPRT devolveu ao paciente a autoestima por melhorar a estética e a fonética, além de manter o espaço até a confecção do aparelho ortodôntico apropriado, sem interferir no crescimento ósseo, sendo importante até a estabilização da DVO, havendo um acompanhamento periódico mensalmente, para posterior tratamento definitivo ${ }^{6}$.

Prabhakar et al. ${ }^{9}$ (2009) descrevem que a desvantagem de uma prótese parcial removível é que a base da prótese acrílica deve cobrir o tecido mole e em alguns casos os ajustes necessários podem ser difíceis nos pacientes jovens. Tal fato não ocorreu no caso clínico em questão, pois o paciente foi bastante colaborativo.

\section{CONCLUSÃO}

Os resultados demonstraram que o tratamento reabilitador com PPRT foi resolutivo para repor os elementos perdidos, atuando na preservação do espaço temporariamente, até a instalação do aparelho ortodôntico apropriado, sem interferir no crescimento ósseo, sendo importante até a estabilização da DVO, restabelecendo estética e função.

\section{REFERÊNCIAS}

1. Silva MCVS, Carreiro AFP, Bonan RF, Carlo HL, Batista AUD. Reabilitação Oclusal com Prótese Parcial Removível Provisória Tipo "Overlay": Relato de Caso. R Bras Cienc Saúde. 2011;15(4):455-60.

2. Bessadet M, Nicolas E, Sochat M, Hennequin M, Veyrune JL. Impact of removable partial denture 
prosthesis on chewing efficiency. J Appl Oral Sci. 2013;21(5):392-6.

3. Pereira CVCA, Soares ARL, Coutinho TCL. Aparelho mantenedor de espaço estético fixo em odontopediatria. Rev Flum Odontol. 2010;16(33):12-4.

4. Srkyl A, Kadithota M. Remote tooth in immediate partial denture: a case report. J Clin Diag Res. 2011;5(6):1318-20.

5. Kamble VD. Rehabilitation of severely worn dentition and partial edentulism by fixed and removable prostheses: a clinical report. Int $\mathbf{J}$ Prosthodont Rest Dent. 2013;3(2):57-61.

6. Shibayama R, Shibayama B, Gennari F H, Saez D, Watanabe E. Próteses totais imediatas convencionais. Rev Odontol Araçatuba. 2006;27(1):67-72.

7. Dantas EM. A importância do restabelecimento da dimensão vertical de oclusão na reabilitação protética. Odonto. 2012;20(40):41-8.

8. Pereira L, Miasato JM. Mantenedor de Espaço Estético-funcional em Odontopediatria. Rev Odontol Univ Cid São Paulo. 2010;22(2):154-62.

9. Prabhakar AR, Sugandhan R, Roopa KB, Akanksha G. Esthetic management of an anterior avulsed tooth: A case report. Int J Clin Pediatr Dent. 2009;2(3):35-8.

10. Abduo J. An innovative prosthesis design for rehabilitation of severely mutilated dentition: a case report. J Adv Prosthodont. 2011;3:37-42.

11. Zachrisson BU, Toreskog S. Esthetic considerations in restoring the traumatized dentition: a biologic approach. In: Andreasen JO, Andreasen FM, Andersson L, eds. Textbook and color atlas of traumatic injuries to the teeth. $4^{\text {th }} \mathrm{ed}$. Munksgaard: Blackwell Inc; 2007:798-813.

12. Bidra AS, Martin JW, Feldman E. Complete denture prosthodontics in children with ectodermal dysplasia: review of principles and techniques. Compend Contin Educ Dent. 2010;31(6):426-44.

13. Marques NCT, Gurgel CV, Fernandes AP, Lima MC, Machado MAAM, Soares S, Oliveira TM. Prosthetic Rehabilitation in Children: An Alternative Clinical Technique. Case Rep Dent. 2013;2013:1-5.

14. Sander HH, Souza EL, Alvim HH, Cornacchia TPM, Lanza LD, Oliveira RR. Projeto próteses provisórias: Quatro anos de contribuição para a qualidade de vida. Arq Odontol. 2011;47(Supl 2):111-3.

15. Dominguez A, Aznar T. Removable prosthesis for preschool children: report of twoo cases. Quintessence Int. 2004;35:397-400.

16. Parisotto TM, Silva CMS, Oliveria CS, Santos MN, Gavião MBD. Prosthetic rehabilitation in a four-year-old child with severe early childhood caries: a case report. J Contemp Dent Pract. 2009;10(2):1-8.

17. Consolaro A. O gene e a epigenética: as características dentárias e maxilares estão relacionadas com fatores ambientais - Ou Os genes não comandam tudo! Ou $\mathrm{O}$ determinismo genético acabou? R Dental Press Ortodon Ortop Facial. 2009;14(6):14-8.

\section{CONFLITO DE INTERESSES}

Os autores declaram não haver conflitos de interesse.

\section{AUTOR PARA CORRESPONDÊNCIA}

Gisely Naura Venâncio

ginaura@gmail.com
Submetido em 11/01/2016 Aceito em 21/01/2016 